\title{
Health and Nutritional Status among Pet Owners and Non-Pet Owners in Kuala Lumpur
}

\author{
Lee Wen $\mathrm{Li}^{1}$, HayatiMohd Yusof ${ }^{1}$, NorSalihah Zakaria ${ }^{1}$, and Asma' $\mathrm{Ali}^{1}$ \\ ${ }^{I}$ (School of Food Science \& Technology, Universiti Malaysia Terengganu, 21030 Kuala Terengganu, Malaysia)
}

\begin{abstract}
The study investigated the effect of pet ownership on the nutritional status and perceived health status among adults. This study involved 160 adult respondents ( 80 pet owners, 80 non-pet owners) recruited in Kuala Lumpur. Respondents completed Lexington-Pet Attachment to Pet Scale (only for pet owners), ShortForm Health Survey-12 (SF-12) and Perceived Stress Scale. The results indicate that body mass index, perceived physical health, perceived mental health, and perceived stress among pet owners were not significantly different than non-pet owners. However, when pet attachment level was considered, it was found that pet owners who were highly bonded to their pets reported better mental health and perceived stress than non-pet owners, but not better perceived physical health $(\mathrm{p}<0.05)$. A correlation test showed no association between pet attachment level and perceived physical health of pet owners $(\rho=0.194, p>0.05)$. Nevertheless, greater attachment to a pet was associated with better perceived mental health $(r=0.336, p<0.05)$ and lower perceived stress $(\rho=-0.412, p<0.05)$. This study revealed that pets positively impact their owners' overall perceived mental health and perceived stress level, but this appears to differ based on the pet attachment level.
\end{abstract}

Keywords -pet ownership, nutritional status, perceived physical health status, perceived mental health status, pet attachment level

\section{INTRODUCTION}

The According to World Health Organization [1], the term healthy is used to describe the state of an individual in the absence of any diseases and illnesses. People often may consider themselves healthy if they are not suffering from any disease. However, the true meaning of the term 'healthy' is far more complicated. An individual with a disease may also be considered healthy to a certain extent according to his ability to build an internal equilibrium that allows him to get the most he can from his life despite the presence of disease [2]. Therefore, health is generally comprised of an integration of psychological, physical, social, environmental and spiritual aspect of individual. An individual may achieve maximal health by living life to its fullest and establishing a state of balance of all dimensions of health.Health status refers to the level of health of an individual, whether subjectively assessed by the individual or by more objective measures. There has been a rise in non-communicable diseases in Malaysia, mostly due to unhealthy urban lifestyles. People suffer from work stress, sedentary lifestyles, and unhealthy eating habits. According to the National Health and Morbidity Survey [3], the most prevalent health issue in Malaysia is cardiovascular disease. The category of health problems ranked second after cardiovascular disease is mental disorders. Mental health problems in Malaysia are expected to increase over the next 20 years [4].Pets are widely known for providing various health benefits to their owners. These days, many therapies incorporate pets for health benefit purposes. Animal-assisted therapy and animal-assisted activities are two examples that animals can enhance or compromise individual's health. There are some suggestions that pet ownership leads to better self-care. Previous studies have shown that pet ownership has favorable effects on cardiovascular risk [5]. One study found that dog owners were healthier due to a less sedentary life and lower blood pressure than non-pet owners [6]. Other than that, cohort studies have shown that people with pets as companions experienced a higher level of physical activity and reduced mortality risk compared to those with no pets[7]. Other benefits to health have also been observed. Studies have found that owning a pet is great for enhance mental health, as pets is good for stress release. One previous study showed that workers tended to have a reduced stress level when they were allowed to bring their pet to work [8]. In addition, pets are able to help fight depression. They are highly therapeutic, explaining the usage of animalassisted therapy by mental health professionals in treating mental disorder patients. By owning a pet, it is not only beneficial to individual with mental disorder but also to everyone especially older adults as pets provide a sense of social support to them. Another study showed that pets can provide older adults with physical contact and comfort and decrease loneliness and depression. McConnell and colleagues found that pet owners reported less loneliness and higher self-esteem than non-pet owners [9]. To date, only two studies have been done in Malaysia to investigate the effect of pet ownership on self-esteem and stress [10,11]. The lack of study into the understanding of pet benefits on the overall perceived health of Malaysians is due to the fact that most studies of 
the health benefits of pet ownership were done in Western countries among Caucasians, primarily dog owners. The extent to which adults from other ethnic groups, especially in Asia, may benefit from owning a pet is unclear. Therefore, current study was done to provide more information on the impact of pet ownership towards health of pet owners in Malaysia. The information obtained may provide an alternative to improve the current health status of Malaysians, as pets may provide benefits for better health, especially mental health.

\section{METHODS}

TheA cross-sectional study was carried out in a study in Kuala Lumpur for a duration of two months from June to September 2015. Based on Yamane [12], a total of 160 respondents (80 pet owners and 80 non-pet owners) were recruited in the study through convenience, purposive, and quota sampling methods. The research instrument used in the present study was a self-administered questionnaire consisting of the Lexington Attachment to Pet Scale [13], short-form health survey (SF-12) [14], and Perceived Stress Scale [15]. Reliability of the questionnaire was tested through a pilot test. Data obtained was analyzed using Statistical Package for the Social Sciences (SPSS) version 20. Normality determination using Kolmogorov-Smirnov test was carried out in data analysis of the study. A descriptive test was used to determine the overall BMI, the pet attachment level, perceived physical and mental health level as well as stress level of the respondents. To make comparisons, the independent T-test or Mann-Whitney test was used to compare BMI, perceived physical, mental health and perceived stress level among pet owners and non-pet owners. The test was also used to compare the perceived physical and mental health between pet owners highly bonded to pets, and non-pet owners. To explore the relationship between degree of bonding and perceived physical and mental health for pet owners, Pearson product-moment correlations or Spearman's rank order correlation were used. In all analyses, results were considered significant with $\mathrm{p}$ equal or less than 0.05 .

\section{RESULTS AND DISCUSSIONS}

\subsection{Sociodemographic of Respondents}

There were 160 participants, including pet owners and non-pet owners. The participants were 46 males (28.8\%) and $114(71.2 \%)$ females. The sample had many more women than men. There were no gender differences in pet attachment according to Herzog [16]. Age of the participants was divided into three groups: 19 to 30 years old; 31 to 50 years old; and 51 to 60 years old. The range age of 19 to 30 years old had the highest frequency at 116 people $(72.5 \%)$, followed by those 31 to 50 years old (23.1\%). This showed that most participants were in the working age group with a median age of 27 years. This group makes up the majority of Malaysia, which has a median age of 27.4 years (Central Intelligence Agency, 2014)[17]. Table 1 shows the sociodemographic profile of the respondents.

\subsection{Pet Ownership}

Pet Ownership Status.

Among pet owners, there were 25 males (31.2\%) and 55 females (68.8\%). There were 20 cat owners (25.0\%), 53 dog owners (66.3\%), and 7 participants who owned small animals such as tortoise and hamsters (8.7\%). Time spent playing with pets was also examined. It was found that there were only 30 pet owners (37.5\%) that play with their pets for less than one hour a day, while another 24 pet owners (30.0\%) play with their pets for one to two hours a day. Fewer pet owners were involved in spending a greater amount of time with their pets. In short, more of the pet owners spent less time with their pets, and probably had less interaction with pets as well as being less dedicated to them. This was probably due to their busy working life, as most of them were a full-time employee. Table 2 shows the pet ownership status of pet owners.

Pet attachment level.

The pet attachment levels of the pet owners were explored through Lexington pet attachment scale, with scores ranging from 33 to 87 in which higher scores indicated stronger attachment. The results showed that the mean pet attachment mean score for 80 pet owners was 66.94 (68.1\%) ( $\mathrm{SD}=11.15)$, considered an average pet attachment level. For the purposes of better comparing the effect of pet ownership among pet owners and non-pet owners, pet attachment level was categorized into three groups, namely below average, average, and above average based on the pet attachment mean score. 41 of pet owners (51.2\%) had a poor pet attachment level, while 25 of the pet owners (31.3\%) had an average pet attachment level and only 14 of the pet owners $(17.5 \%)$ had a strong pet attachment level. Overall, there was a higher proportion of pet owners poorly attached to their pet, and they were predicted to receive fewer of the health benefits provided by pets. Table 3 shows categories of pet attachment level. 


\subsection{Comparison between Pet Owners and Non-Pet Owners 3.3.1 Body Mass Index.}

Based on the results, the median of body mass index for pet owners was $21.33(4.42) \mathrm{kg} / \mathrm{m} 2$ while median of body mass index for non-pet owners was 21.16 (3.76) $\mathrm{kg} / \mathrm{m} 2$. Based on the body mass index classification analysis, both pet owners and non-pet owners were overall considered to place in the healthy categories. In order to answer research question about the possible difference of body mass index among pet owners and non-pet owners, the Mann-Whitney test statistic was employed. The results as indicated in Table 4 show no difference in the median body mass index between groups $(\mathrm{p}>0.05)$. A study done by Anderson et al. [5] reached similar findings, with no difference in body mass index between groups of overweight pet owners and non-pet owners. Despite no difference in the body mass index between groups, they found that pet owners had significantly more exercise than non-pet owners. Another study by Parslow\&Jorm[18] involved randomly selected Australian pet owners (dogs, cats, birds or fish) and non-pet owners. They also found that pet owners $(26.85 \mathrm{~kg} / \mathrm{m} 2)$ had no difference in body mass index with non-pet owners $(26.36 \mathrm{~kg} / \mathrm{m} 2)$. Ideally, pet owners might have a healthier body mass index than non-pet owners due pet had a positive influence on the physical activity level of its owners. However, this may be only applicable to dog owners. Other than dog ownership, it was reported that cat or other types of pet ownership had no association with physical activity. Even with dog ownership, owners might not gain benefits if they are not the ones walking with and interacting with their dog.

\subsubsection{Perceived physical health and mental health of the respondents.}

Pet owners and non-pet owners' perceived physical health and mental health were measured using the physical component summary scores and mental component summary scores of the short-form health survey-12 (SF-12). In terms of perceived physical health, the total scores for the physical component summary of shortform health survey-12 ranged from 21.9 to 63.5 , in which higher scores indicate better physical health. Based on the results of the present study, it can be reported that pet owners had a median physical component scores of 52.45 (11.00). While for non-pet owners had 51.65 (10.20). At $\alpha=0.05$ level of significance, there was no difference in the physical component score of both groups (Table 5). In terms of perceived mental health, the total scores for the mental component summary of short-form health survey-12 ranged from 28.2 to 63.7 , with higher scores indicating better mental health. The result indicated that pet owners had mean mental component scores of 46.68 (6.86) while for non-pet owners was 45.58 (8.18). At $\alpha=0.05$ level of significance, there was no difference in the mental component score (Table 6) between group of pet owners and non-pet owners. The present results on the perceived physical health and mental health contradict the findings that supported the idea of better self-perception of physical and psychological health in pet owners than non-pet owners, as reported by Serpell[19] and Serpell[20]. The insignificance of the present finding might be due to the majority of respondents in both groups were generally healthy younger adults without any known diseases. Besides, for those who own pets, their health perceptions may also be influenced by pet attachment level [20]. Since a majority of the pet owners had poor pet attachment, it can be said that real health conditions may be the factor influencing the perception on their own physical health and mental health, instead of the effect of pet ownership. The other possible reason for contradictory results was the different races and ethnicities involved in the study. This is because difference races or ethnicities may have different susceptibility to disease and self-identification, as well as own perception of health [21]. In addition, pets are perceived to serve different roles and functions by different races or ethnicities [22].

\subsubsection{Perceived stress.}

Pet owners and non-pet owners' perceived stress level were measured using the Perceived Stress Scale. The total scores for the perceived stress assessment ranged from 2 to 33 for all respondents, with higher scores relating to higher levels of perceived stress. The result in the present study revealed that pet owners had a median stress assessment scores of 15.0 (9.0) while for non-pet owners was $17.0(9.0)$. The details are shown in the Table 7. Pet owners did score slightly lower than the non-pet owners in stress assessment, indicating that pet owners had a better perceived stress level than non-pet owners, even though the difference is not statistically significant ( $p>0.05)$. The results of the present study contradicted previous findings $[11,23,24]$ which stated that pet ownership was found to have an association with lower perceived stress among pet owners. The differences between in the present findings and previous findings may be due to several reasons. One possible reason was that the majority of pet owners involved in the present study had poor pet attachment. Most of their pets were taken care by other household members and they spent less time together. Therefore, they experienced less interaction with their pets. As a result, they were unable to get benefit from their pets through the support given which able to boost their positive feeling. Another reason was probably due to difference in methodology. Previous studies often consider aspects of social support from family, friends, or pets which more accurately predicted the perceived stress in pet owners. Lack of social support from either people or pets could lead to 
greater perceived stress. On the other hand, people with social support fulfilment from either people or pets were more likely to experience less perceived stress [23].

\subsection{Comparison between Highly Bonded Pet Owners and Non-Pet Owners 3.4.1 Perceived Physical Health and Mental Health.}

Although the hypothesis on the difference between pet owners and non-pet owners in perceived physical and mental health was rejected, the level of pet attachment in perceived physical and mental health was explored. In this case, only pet owners who were highly bonded to their pets were compared with non-pet owners in their own perception of physical and mental health. Table 8 shows that there was no significant difference in the median physical component score $(p>0.05)$ between groups, although a higher score was seen in pet owners. However, for the perceived mental health as shown in Table 9, highly attached pet owners did score significantly higher in mental than non-pet owners $(52.76 \mathrm{vs} 45.58 ; \mathrm{p}=0.002)$. This indicates that pet owners who were highly attached to pet had a significantly better perceived mental health than non-pet owners, but not in physical health. The insignificant result in perceived physical health could be possibly due to factor of body mass index. Individuals with a high body mass index, especially those with overweight or obesity problems, might have a lower self-esteem and greater limitations on certain activities. In the present study, about $32.4 \%$ of pet owners had a weight problem. Koivusilta\&Ojanlatva[25] found that a large body mass index in pet owners could became a factor that negatively affects the association of pet ownership with perceived physical health. The negative effects of large body mass index overpowered the positive effect of pet ownership on the owners' perception on physical health. However, in the case of perceived mental health, the present results are supported by the study done by Serpell [19] which showed better self-perception psychological health in pet owners than non-pet owners. Pet owners with a high body mass index may have a better self-perception on their mental health due to the effect of pet ownership. This was because they were being more attached to their pets, as they had a less strong network of social support fulfilment from human [26]. Pet owners who are highly attached to pets would consider their pets as part of the family and might receive much love, support and feel valued from their interaction with pets, indirectly helping to positively shape owners' identities.

\subsubsection{Perceived stress.}

Since there was no significant difference in perceived stress between pet owners and non-pet owners, further investigation was done to include the aspect of pet attachment in the determination of the effect of pet ownership on perceived stress. Table 10 shows that the median (IQR) of stress level in pet owners who were highly bonded to pet [12.5 (5.0)] was significantly lower $(\mathrm{p}<0.05)$ than non-pet owners [17.0 (9.0)]. This time, the present findings were fully supported by previous studies by Shoda et al. [24] and Lee \& Chai [11] where lower perceived stress level in pet owners when the aspect of pet attachment was being considered. There was less perceived stress in pet owners when their social needs fulfilment came from pets. Pet owners who scored highly in the pet attachment scale in the present study were assumed to have greater social support from their pets. Non-evaluative supports provided by pets were able to boost positive feelings in pet owners, helping owners to improve their stress coping abilities and experience lower levels of perceived stress [27].

\subsection{Correlation between Pet Attachment Level and Perceived Physical Health, Mental Health and Perceived Stress}

3.5.1 Relation between pet attachment level and perceived physical health.

In order to test for the relation between the pet attachment and perceived physical health for pet owners, the Spearman correlation coefficient was used. As shown in Table 11, the intensity of attachment to pet had no correlation to the perceived physical health $(p>0.05)$. From the result, it was indicated that the degree of bonding to pets had no influence on pet owners' perceived physical health. The results of the present study are contradicted with the findings from a previous study [28]. The study found that higher intensity of attachment to pets was positively correlated with greater perceived physical and mental health benefits, as pet owners believed that they had better health. However, the study only focused on dog ownership and the sample was largely recruited from dog owner associations in which their respondents were highly committed to their pets. This may have resulted in biases in their finding. Also, the differences between the present study and previous findings may be due to the factor of body mass index. Koivusilta\&Ojanlatva[25] suggested that a large body mass index in pet owners could became a factor that negatively affects the association of pet ownership with perceived physical health even if the pet owners were highly attached to pets.

\subsubsection{Relation between pet attachment level and perceived mental health and perceived stress.}

In order to test for the relation between the pet attachment and perceived mental health for pet owners, Pearson correlation coefficient was used. Based on the analysis shown in Table 11, there was a weak linear correlation between perceived mental health and pet attachment level $(r=0.336, p<0.05)$. This indicated that 
the stronger the attachment to pet, the better the perceived mental health in pet owners. A similar positive result was found in the relationship between pet attachment and perceived stress. Based on the Pearson correlation test, a statistically significant negative week correlation was found between perceived stress and pet attachment level $(\rho=-0.412, p<0.05)$. The findings indicate that the more pet owners are attached to their pet, the lower the level of perceived stress. Both of the findings suggest that pet owners do benefit from their pets with better perceived mental health and lower perceived stress when highly attached to their pets. This finding is consistent with a study by Budge et al. [29], as pet owners who being more attached to pets had better well-being as well as less anxiety and distress. The possible explanation was that with stronger pet attachment, pet owners were more likely to ascribe humanlike emotions to their pets and obtain additional social support from their pets in additions from their family and friends. Social support may serve as a buffer against stressful situations.Antonacopoulos\&Pychyl[30] also found that lower stress levels in pet owners were due to higher amounts of social support when a high level of anthropomorphism was attributed to pets.

\section{CONCLUSION}

In general, pet ownership had a positive impact on pet owners' health status. The present findings show that body mass index, perceived physical health, perceived mental health and perceived stress among pet owners were not significantly better than those of non-pet owners. However, when pet attachment level was considered, results showed that pet owners who are highly bonded to their pets did have a significantly better perceived mental health and perceived stress than non-pet owners, but not better perceived physical health. Based on the findings, it may be suggested that pet owners do not necessarily gain health benefits by owning pets. It all depends on how the pet owners viewed their pets and how strong they attached to pets. Pet owners who are highly attached to pets would consider their pets as part of the family. They might receive much love and mental support and feel valued from their interactions with pets, which indirectly helps to positively shape their identities. The correlation test showed no association between pet attachment level and perceived physical health of pet owners $(\rho=0.194, p>0.05)$. Nevertheless, greater attachment to a pet was shown to be positively associated with better perceived mental health $(r=0.336, p<0.05)$ and lower perceived stress $(\rho=-0.412, p<$ 0.05). With stronger pet attachment, pet owners were more likely to ascribe humanlike emotions toward their pets and obtain additional social support from their pets in addition to family and friends, which may serve as a buffer against stressful situations. In a nutshell, owning pets did provide some health benefits to pet owners, especially in terms of perceived mental health and perceived stress if there was high attachment to the pets.

There are several limitations to the present study. Firstly, the method chosen was convenience sampling method, in which most of the respondents were younger adults with a median age of 27 years old for both groups of pet owners and non-pet owners. Their profile might not be representative of other age group pet owners. Apart from that, the respondents involved also consisted of a high proportion of Chinese respondents and dog owners, as well those with poor attachments to their pets. These may cause bias in results, which may not be generalizable to other ethnic groups. Second, the health status of the respondents was not measured but purely relied on self-reporting. There was no adjustment for confounding factors such as ethnicity, education level, and marital status in the present study. Those factors also might limit the interpretation of the results.

Given the limitations of the present study, future study may try cover a wider range of age to understand better on different pet owners in variety of age groups. Also, future study may investigate each type of pet separately, as different types of pets have different influences on humans. Besides, to obtain more replicable and specific findings, subsequent studies may include the collection of biochemical data of pet owners such as blood pressure readings, cholesterol levels and blood glucose levels in order to explore the effect of pet ownership on blood pressure level and cardiovascular risks of pet owners. Finally, further studies may examine pet owners and non-pet owners from a variety of dimensions such as socioeconomic status and religious views on pets, so that the health benefits of owning a pet by all sorts of individuals can be fully understood.

\section{REFERENCES}

[1] World Health Organization, Global Status Report on Non-communicable Diseases 2010,Geneva: World Health Organization, 2011.

[2] N. Sartorius, The meanings of health and its promotion, Croatian Medical Journal 47(4), $2006,662-664$.

[3] National Health and Morbidity Survey, Fact Sheet 2011, Ministry of Health Malaysia, 2011, from www.moh.gov.my [Retrieved Aug 25 2015].

[4] More mental health issues, The Star, 2014, from http://www.thestar.com.my [Retrieved Jun 5 2015].

[5] W.P. Anderson, C.M. Reid and G.L. Jennings, Pet ownership and risk factors for cardiovascular disease, The Medical Journal of Australia 157(5), 1992, 298-301. 
[6] K. Arhant-Sudhir, R. Arhant-Sudhir,andK. Sudhir, Pet ownership and cardiovascular risk reduction: Supporting evidence, conflicting data and underlying mechanisms, Clinical and Experimental Pharmacology and Physiology 38, 2011, 734-738.

[7] R.F. Gillum, and T.O. Obisesan, Living with companion animals, physical activity and mortality in a U.S. national cohort,International Journal of Environmental Research and Public Health7, 2010, 24522459.

[8] M. Wells, and R. Perrine, Critters in the cube farm: perceived psychological and organizational effects of pets in the workplace,Journal of Occupational Health Psychology 6(1), 2001, 81-87.

[9] A.R. McConnell, C.M. Brown, T.M. Shoda, L.E. Stayton, andC.E. Martin, Friends with benefits: On the positive consequences of pet ownership,Journal of Personality and Social Psychology 101(6), 2011, 1239-1252.

[10] C.S. Kuan, andT.S. Jin, The effects of owning a pet on self-esteem and self-efficacy of Malaysian pet owners,Sunway Academic Journal 2, 2005, 85-91.

[11] V.K. Lee, andM.S. Chai, Dog ownership, perceived social supports and stress among university students,American Journal of Applied Psychology 4(3), 2015, 45-50.

[12] T. Yamane, Statistics: An Introductory Analysis(New York: Harper and Row, 1967).

[13] D. Anderson, Assessing the human-animal bond: A compendium of actual measures (West Lafayette, Ind: Purdue University Press, 2007)

[14] J.E. Ware, M.M.A. Kosinski, andS.D. Keller, A 12-item short-form health survey: Construction of scales and preliminary tests of reliability and validity, Med Care 34(3), 1996, 220-233.

[15] S. Cohen, and G. Williamson, Perceived Stress in a Probability Sample of the United States in S. Spacapan, and S. Oskamp (Eds.),The Social Psychology of Health (Newbury Park, CA: Sage, 1988) 6164.

[16] H.A. Herzog, Gender Differences in Human-Animal Interactions: A Review,Anthrozoo 20, 2007, 17-21.

[17] Central Intelligence Agency, The world factbook - Malaysia [online], 2014, from: http://www.indexmundi.com [Retrieved May 23 2015].

[18] R.A. Parslow, andA.F. Jorm, The impact of pet ownership on health and health service use: Results from a community sample of Australians aged 40 to 44 years,Anthrozoos 16(1), 2003, 43-56.

[19] J.A. Serpell, Beneficial effects of pet ownership on some aspects of human health and behavior,Journal of the Royal Society of Medicine 84, 1991,717-720.

[20] J.A. Serpell, Evidence for an association between pet behavior and owner attachment levels,Applied Animal Behavior Science 47, 1996,49-60.

[21] R. Bulatao, Understanding racial and ethnic differences in health in late life a research agenda (Washington, DC: National Academies Press, 2004).

[22] C. Risley-Curtiss, L.C. Holley, andS. Wolf, The animal-human bond and ethnic diversity,Social Work 51(3), 2006, 257-268.

[23] J. Kraus, Stress in Pet Owners and Non-Pet Owners [online], 2012, from http://63.128.9.244/document.doc?id=120 [Retrieved July 20 2015].

[24] T.M. Shoda, L.E. Stayton, andC.E. Martin, Friends with benefits: on the positive consequences of pet ownership, Journal of Personality and Social Psychology 101(6), 2011, 1239-1252.

[25] L.K. Koivusilta and A. Ojanlantva, To have or not to have a pet for better health? PLoS ONE 1(1), 2006, e109.

[26] M.B. Stephens, C.C. Wilson, J.L. Goodie, F.E. Netting, C.H. Olsen andC.G. Byers, Health perceptions and levels of attachment: Owners and pets exercising together,The Journal of the American Board of Family Medicine 25(6), 2012, 923-926.

[27] K. Allen, J. Blascovich andW. Mendes, Cardiovascular reactivity and the presence of pets, friends, and spouses: The truth about cats and dogs,Psychosomatic Medicine 64,2002, 727-739.

[28] G. Andreaseen, L.C. Stenvold and F.W. Rudmin, "My dog is my best friend": health benefits of emotional attachment to a pet dog, Psychology \& Society 5(2), 2013, 6-23.

[29] C. Budge, J. Spicer, B. Jones andR. St. George, Health correlates of compatibility and attachment in human-companion animal relationships,Society and Animals 6(3), 1998, 219-234.

[30] N. Antonacopoulosand T.A. Pychyl, An examination of the relations between social support, anthropomorphism and stress among dog owners, Anthrozoös 21(2), 2008, 139-152. 
Tables Table 1 Respondents' Socio-Demographic Profile

\begin{tabular}{|c|c|c|c|}
\hline Parameter & $\begin{array}{l}\text { With Pets } \\
\text { n }(\%) \\
(\mathbf{n}=\mathbf{8 0})\end{array}$ & $\begin{array}{l}\text { Without Pets } \\
\text { n }(\%) \\
(\mathrm{n}=\mathbf{8 0})\end{array}$ & $\begin{array}{l}\text { Total Sample } \\
\text { n }(\%)\end{array}$ \\
\hline \multicolumn{4}{|l|}{ Sex } \\
\hline Male & $25(31.2 \%)$ & $21(26.2 \%)$ & $46(28.8 \%)$ \\
\hline Female & $55(68.8 \%)$ & $59(73.8 \%)$ & $114(71.2 \%)$ \\
\hline \multicolumn{4}{|l|}{ Age (Years old) } \\
\hline $19-30$ & $60(75.0 \%)$ & $56(70.0 \%)$ & $116(72.5 \%)$ \\
\hline $31-50$ & $14(17.5 \%)$ & $23(28.8 \%)$ & $37(23.1 \%)$ \\
\hline $51-60$ & $6(7.5 \%)$ & $1(1.2 \%)$ & $7(4.4 \%)$ \\
\hline \multicolumn{4}{|l|}{ Races } \\
\hline Malay & $17(21.2 \%)$ & $14(17.5 \%)$ & $31(19.4 \%)$ \\
\hline Chinese & $57(71.2 \%)$ & $60(75.0 \%)$ & $117(73.1 \%)$ \\
\hline Indian & $6(7.5 \%)$ & $6(7.5 \%)$ & $12(7.5 \%)$ \\
\hline \multicolumn{4}{|l|}{ Marital status } \\
\hline Single & $58(72.5 \%)$ & $56(70.0 \%)$ & $114(71.2 \%)$ \\
\hline Married & $21(26.2 \%)$ & $23(28.8 \%)$ & $44(27.5 \%)$ \\
\hline Divorced/ Widowed & $1(1.2 \%)$ & $1(1.2 \%)$ & $2(1.2 \%)$ \\
\hline \multicolumn{4}{|l|}{ Education } \\
\hline Primary school & $2(2.5 \%)$ & $0(0.0 \%)$ & $2(1.2 \%)$ \\
\hline Secondary school & $10(12.5 \%)$ & $12(15.0 \%)$ & $22(13.8 \%)$ \\
\hline University/ College & $68(85.0 \%)$ & $68(85.0 \%)$ & $136(85.0 \%)$ \\
\hline \multicolumn{4}{|l|}{ Working status } \\
\hline Full-time & $54(67.5 \%)$ & $51(63.8 \%)$ & $105(65.6 \%)$ \\
\hline Part-time & $6(7.5 \%)$ & $3(3.8 \%)$ & $9(5.6 \%)$ \\
\hline Student & $15(18.8 \%)$ & $18(22.5 \%)$ & $33(20.6 \%)$ \\
\hline No working & $3(3.8)$ & $8(10.0 \%)$ & $11(6.9 \%)$ \\
\hline Retired & $2(2.5 \%)$ & $0(0.0 \%)$ & $2(1.2 \%)$ \\
\hline
\end{tabular}

Table 2 Pet Ownership Status $(\mathrm{n}=80)$

\begin{tabular}{|l|l|l|}
\hline Parameter & Frequency (n) & Percentage (\%) \\
\hline Pet type & & \\
\hline Cat & 20 & 25.0 \\
\hline Dog & 53 & 66.3 \\
\hline Small animals & 7 & 8.7 \\
\hline Years owned pet & & \\
\hline Less than one year & 4 & 5.0 \\
\hline $1-5$ years & 37 & 46.3 \\
\hline $6-10$ years & 22 & 27.5 \\
\hline More than 10 years & 17 & 21.2 \\
\hline Playing times with pet & & \\
\hline$<1$ hour & 30 & 37.5 \\
\hline $1-2$ hours & 24 & 30.0 \\
\hline $2-2.5$ hours & 18 & 22.5 \\
\hline $2.5-3$ hours & 3 & 3.8 \\
\hline $3-3.5$ hours & 1 & 1.2 \\
\hline $3.5-4$ hours & 1 & 1.2 \\
\hline$>4$ hours & 3 & 3.8 \\
\hline
\end{tabular}

Table 3 Pet Attachment Level

\begin{tabular}{|l|l|l|}
\hline & Frequency $(\mathbf{n})$ & Percentage $(\%)$ \\
\hline Pet attachment level $(\mathbf{n}=\mathbf{8 0})$ & & \\
\hline Below average $(<55.8)$ & 41 & 51.2 \\
\hline Average $(55.8-78.1)$ & 25 & 31.3 \\
\hline Above average $(>78.1)$ & 14 & 17.5 \\
\hline
\end{tabular}


Table 4 Comparison of BMI between Pet Owners and Non-Pet Owners

\begin{tabular}{|l|l|l|l|l|}
\hline Variables & $\begin{array}{l}\text { Pet owners } \\
(\mathbf{n = 8 0}) \\
\text { median (IQR) }\end{array}$ & $\begin{array}{l}\text { Non-pet owners } \\
(\mathbf{n = 8 0}) \\
\text { median (IQR) }\end{array}$ & $\boldsymbol{U}$ value \\
\hline BMI & $21.332(4.420)$ & $21.156(3.760)$ & 2846.000 & 0.227 \\
\hline
\end{tabular}

Table 5 Comparison of Perceived Physical Health between Pet Owners and Non-Pet Owners

\begin{tabular}{|l|l|l|l|l|}
\hline Variables & $\begin{array}{l}\text { Pet owners } \\
(\mathbf{n = 8 0 )} \\
\text { median (IQR) }\end{array}$ & $\begin{array}{l}\text { Non-pet owners } \\
(\mathbf{n = 8 0}) \\
\text { median (IQR) }\end{array}$ & $\boldsymbol{U}$ value \\
\hline PCS & $52.45(11.00)$ & $51.65(10.20)$ & 3068.00 & 0.652 \\
\hline
\end{tabular}
p> 0.05 indicates not significantly different by Mann-Whitney test

Note: Physical component scores ranged from 0 to 100, 0 being the worst while 100 being the best.

Table 6 Comparison of Perceived Mental Health between Pet Owners and Non-Pet Owners

\begin{tabular}{|l|l|l|l|l|l|}
\hline Variables & $\begin{array}{l}\text { Pet owners } \\
(\mathbf{n = 8 0}) \\
\text { mean (SD) }\end{array}$ & $\begin{array}{l}\text { Non-pet } \\
\text { owners } \\
(\mathbf{n = 8 0}) \\
\text { mean } \\
(\mathrm{SD})\end{array}$ & $\begin{array}{l}\text { Mean diff. } \\
(\mathbf{9 5 \%} \text { CI) }\end{array}$ & $\begin{array}{l}\boldsymbol{t} \text {-statistic } \\
(\mathbf{d f})\end{array}$ & $\boldsymbol{p}$ value \\
& $\begin{array}{l}45.57 \\
(8.18)\end{array}$ & $1.10(-1.25,3.46)$ & $0.927(158)$ & 0.356 \\
\hline MCS & 46.68 & & & \\
\hline
\end{tabular}

p> 0.05 indicates not significantly different by Independent $t$ test

Note: Mental component scores ranged from 0 to 100, 0 being the worst while 100 being thebest.

Table 7 Comparison of Perceived Stress Level between Pet Owners and Non-Pet Owners

\begin{tabular}{|l|l|l|l|l|}
\hline Variables & $\begin{array}{l}\text { Pet owners } \\
(\mathbf{n = 8 0}) \\
\text { median }(\mathrm{IQR})\end{array}$ & $\begin{array}{l}\text { Non-pet owners } \\
(\mathbf{n = 8 0}) \\
\text { median (IQR) }\end{array}$ & $\boldsymbol{U}$ & $\boldsymbol{p}$ value* \\
\hline $\begin{array}{l}\text { Stress } \\
\text { Assessment }\end{array}$ & $15.00(9.00)$ & $17.00(9.00)$ & 3193.50 & 0.982 \\
\hline
\end{tabular}

$p>0.05$ indicates not significantly different by Mann-Whitney test

Note: Scores on perceived stress ranged from 0 to 40 , with higher scores indicating greater stress.

Table 8 Comparison of Perceived Physical Health between Pet Owners Who Are Highly Bonded to Pets and

\begin{tabular}{|l|l|l|l|l|}
\hline Variables & $\begin{array}{l}\text { Pet owners } \\
(\mathbf{n = 1 4 )} \\
\text { median }(\mathrm{IQR})\end{array}$ & $\begin{array}{l}\text { Non-pet owners } \\
(\mathbf{n = 8 0}) \\
\text { median (IQR) }\end{array}$ & $\boldsymbol{U}$ & $\boldsymbol{p}$ value \\
\hline PCS & $53.35(5.30)$ & $51.65(10.20)$ & 446.50 & 0.228 \\
\hline
\end{tabular}

Table 9 Comparison of Perceived Mental Health between Pet Owners Who Are Highly Bonded to Pet and NonPet Owners

\begin{tabular}{|l|l|l|l|l|l|}
\hline Variables & $\begin{array}{l}\text { Pet owners } \\
(\mathbf{n = 1 4 )} \\
\text { mean (SD) }\end{array}$ & $\begin{array}{l}\text { Non-pet } \\
\text { owners } \\
(\mathbf{n = 8 0} \\
\text { mean (SD) }\end{array}$ & $\begin{array}{l}\text { Mean diff. } \\
(\mathbf{9 5 \%} \text { CI) }\end{array}$ & $\begin{array}{l}\boldsymbol{t} \text {-statistic } \\
(\mathbf{d f})\end{array}$ & $\boldsymbol{p}$ value \\
\hline MCS & 52.757 & $\begin{array}{l}45.575 \\
(8.182)\end{array}$ & $\begin{array}{l}7.182 \quad(2.638, \\
11.726)\end{array}$ & $3.139(92)$ & $0.002 *$ \\
& $(5.885)$ & $* p<0.05$ indicates significantly different by Independent $t$ test \\
\hline
\end{tabular}


Table 10 Comparison of Perceived Stress Level between Highly Attached Pet Owners and Non-Pet Owners

\begin{tabular}{|l|l|l|l|l|}
\hline Variables & $\begin{array}{l}\text { Pet owners } \\
(\mathrm{n}=14) \\
\text { median (IQR) }\end{array}$ & $\begin{array}{l}\text { Non-pet owners } \\
(\mathrm{n}=80) \\
\text { median (IQR) }\end{array}$ & $U$ & $p$ value \\
\hline $\begin{array}{l}\text { Stress } \\
\text { Assessment }\end{array}$ & $12.500(5.000)$ & $17.000(9.000)$ & 331.000 & $0.015^{*}$ \\
\hline
\end{tabular}

Table 11 Relation between Degrees of Bonding and Perceived Physical Health, Mental Health and Perceived Stress

\begin{tabular}{|c|c|c|}
\hline Correlation (r) & Degree of bonding & Sig. (2-tailed) \\
\hline Perceived physical health & 0.194 & 0.085 \\
\hline Perceived mental health & $0.336^{* *}$ & 0.000 \\
\hline Perceived stress & $-0.412^{* *}$ & 0.000 \\
\hline
\end{tabular}

$* *$ Correlation is significant at the 0.05 level. 